Documentation et bibliothèques

DOCUMENTATION BIBLIOTHEQUES

\title{
Jeunes néo-québécois et lecture
}

Young Neo-Quebecois and Reading

Los jóvenes neoquebequenses y la lectura

\section{Hélène Roy}

Volume 31, numéro 3, juillet-septembre 1985

URI : https://id.erudit.org/iderudit/1052776ar

DOI : https://doi.org/10.7202/1052776ar

Aller au sommaire du numéro

\section{Éditeur(s)}

Association pour l'avancement des sciences et des techniques de la documentation (ASTED)

ISSN

0315-2340 (imprimé)

2291-8949 (numérique)

Découvrir la revue

Citer cet article

Roy, H. (1985). Jeunes néo-québécois et lecture. Documentation et bibliothèques, 31(3), 93-99. https://doi.org/10.7202/1052776ar
Résumé de l'article

À partir de données cueillies et élaborées grâce à la collaboration des élèves de l'École Enfant-Soleil de Saint-Laurent, une ville comptant une quarantaine de groupes ethniques, l'auteur identifie les goûts littéraires des jeunes néo-québécois, mesure leurs besoins et leurs attentes et les compare à ceux des enfants d'origine québécoise et canadienne.
Tous droits réservés (C) Association pour l'avancement des sciences et des techniques de la documentation (ASTED), 1985
Ce document est protégé par la loi sur le droit d'auteur. L'utilisation des services d'Érudit (y compris la reproduction) est assujettie à sa politique d'utilisation que vous pouvez consulter en ligne.

https://apropos.erudit.org/fr/usagers/politique-dutilisation/ 


\title{
Jeunes néo-québécois et lecture
}

\author{
Hélène Roy* \\ Université McGill
}

A partir de données cueillies et élaborées grâce à la collaboration des élèves de l'École Enfant-Soleil de Saint-Laurent, une ville comptant une quarantaine de groupes ethniques, l'auteur identifie les goûts littéraires des jeunes néo-québécois, mesure leurs besoins et leurs attentes et les compare à ceux des enfants d'origine québécoise et canadienne.

\section{Young Neo-Quebecois and Reading}

From data collected and prepared with the cooperation of students from the school EnfantSoleil in Saint-Laurent, a city which counts about forty ethnic groups, the author identifies the literary tastes of young Neo-Quebecois, weighs their needs and expectations, and compares them to those of children born in Quebec and Canada.

\section{Los jóvenes neoquebequenses y la lectura}

A partir de datos recogidos y elaborados con la colaboración de los alumnos de la escuela "EnfantSoleil" de Saint-Laurent, una ciudad con unos cuaranta grupos étnicos, el autor identifica las preferencias literarias de los jóvenes neoquebequenses, mide sus necesidades y sus expectaciones, y las compara con las de los jóvenes de origen quebequense y canadiense.
Ville Saint-Laurent, sur l'île de Montréal, regroupe des personnes d'une quarantaine de groupes ethniques. Les statistiques de la Commission scolaire Sainte-Croix révèlent qu'en 1984/1985 la Commission comptait, au niveau primaire, des enfants de 39 origines nationales différentes. Selon ces données, les jeunes néo-québécois composaient près de $27 \%$ des effectifs de l'ensemble des écoles primaires de la Commission scolaire et à Saint-Laurent ce pourcentage s'établissait à $31 \%$. Une telle diversité culturelle offrait l'occasion idéale de mesurer les perceptions des jeunes immigrés face à leurs bibliothèques publique et scolaire.

L'étude effectuée dans le cadre de la maîtrise en bibliothéconomie à l'Université McGill, porte sur une seule école, l'École Enfant-Soleil, située à environ un kilomètre de la Bibliothèque municipale de Saint-Laurent. Le choix de cet établissement s'est imposé en raison de sa population hétérogène : plus de $80 \%$ des jeunes appartiennent à des familles néo-québécoises. II ne s'agissait donc pas d'un échantillon aléatoire. Il ne s'agissait pas non plus d'une recherche scientifique comportant une hypothèse à vérifier. Elle serait plutôt exploratoire et descriptive. Le but de la recherche était la mesure des besoins et des goûts des jeunes néoquébécois ainsi que de leurs attentes face à la bibliothèque municipale et face à la bibliothèque de leur école. J'ai aussi tenté d'établir une corrélation entre le pays d'origine et les sujets d'intérêt des enfants.

Les variables retenues sont le sexe, l'âge et le pays d'origine des jeunes et de leurs parents, la langue parlée à la maison et le niveau de scolarité. Je me suis aussi intéressée aux activités domestiques des enfants afin d'évaluer la place de la lecture parmi leurs loisirs, à leurs sujets d'intérêt, à la façon dont ils se procurent les livres et aux raisons qui les font fréquenter ou négliger la bibliothèque municipale. Je me suis également attardée à mesurer leur degré de satisfaction visà-vis de la bibliothèque municipale et la bibliothèque scolaire, du type de livres offerts, de la qualité du service et de l'atmosphère qui y règne. La recherche a de plus permis d'identifier les préférences des élèves du $2^{\mathrm{e}}$ cycle en matière de lecture de langues désirées. 
Pour ne pas créer de dissension dans les groupes, j'ai distribué le questionnaire à tous les écoliers québécois et néo-québécois du $2 \mathrm{e}$ cycle, 191 au total, répartis dans trois groupes de $4 \mathrm{e}$ année, trois groupes de $5^{\mathrm{e}}$ et deux groupes de $6^{\mathrm{e}}$ année. La question numéro $3 \mathrm{~m}$ 'a permis d'identifier le pays d'origine de l'enfant et de ses parents.

Le questionnaire comprenait 26 questions. J'ai opté pour des questions à choix multiples auxquelles les enfants pouvaient répondre sans difficultés. Ils avaient la possibilité, à plusieurs reprises, d'encercler plus d'une réponse. En plus des choix multiples, nombre de questions étaient ouvertes, laissant ainsi place aux commentaires.

Le questionnaire fut imprimé uniquement en français sur quatre feuilles dont trois recto verso. Les questions respectaient un ordre logique : données personnelles ( 1 à 4 ), habitudes de lecture et intérêts particuliers (5 à 9 ), bibliothèques municipale et scolaire (10 à 17) et suggestions (18 à 20).

Le pré-test fut effectué le 3 octobre 1984. Au total, soixante-treize questionnaires furent distribués dans trois classes: une de $4^{\mathrm{e}}$ année, une de $5^{\mathrm{e}}$ et une de $6^{\mathrm{e}}$. Les enfants répondaient à mesure que je lisais les questions. Ils pouvaient ainsi se faire expliquer ce qu'ils ne comprenaient pas. $\mathrm{J}$ 'avais précisé qu'ils étaient libres de répondre ou non et que ce jeu questionnaire ne constituait pas un travail scolaire. Le taux de participation fut exceptionnel $(100 \%)$ et tous les questionnaires ont pu être analysés. L'analyse des données du pré-test a permis de vérifier la clarté des questions. Aucune modification majeure ne fut apportée à la suite du pré-test. J'ai seulement reformulé une question afin d'en faciliter la compilation.

La cueillette des données eut lieu une semaine plus tard, le 11 octobre 1984. Elle se fit dans cinq classes du $2^{e}$ cycle non visitées au moment du pré-test, deux classes de $4{ }^{\mathrm{e}}$, deux de $5^{\mathrm{e}}$ et une de $6 \mathrm{e}$. Cent dix-huit élèves complétèrent le questionnaire. Tout comme lors du pré-test, le taux de réponse fut très élevé $(100 \%)$ et tous les questionnaires ont pu être analysés. Cependant, la qualité des réponses fut moindre en raison peut-être de l'insistance mise sur la liberté de répondre ou non aux questions. Par ailleurs, au moment de la cueillette des données, certains enfants ont posé des questions qui ont mené droit à des modifications du questionnaire. Ainsi, il fut nécessaire d'ajouter la catégorie "des fois" à une question dont les deux possibilités de réponses étaient "oui» ou «non».

Le seul contrôle effectué fut celui du niveau d'études. Afin de respecter l'anonymat des répondants, j'avais même suggéré de ne pas écrire son nom sur le questionnaire. Quelques enfants l'ont quand même fait.

Comme je l'ai déjà précisé, le groupe étudié est celui des élèves de $4 \mathrm{e}$ à $6^{\mathrm{e}}$ année de l'École primaire Enfant-Soleil. Deux raisons justifient ce

choix: 1) la majorité des enfants étaient passés en classe d'accueil et savaient lire et écrire le français, 2) les enfants connaissaient la bibliothèque de leur école et, également, la bibliothèque municipale, car l'école y organise une visite chaque année.

La compilation des données selon le sexe, le niveau d'études, l'âge, le pays d'origine et la langue parlée à la maison a permis l'identification de l'échantillonnage. Le groupe analysé comptait soixante-et-une filles et cinquante garçons. Sept répondants $n$ 'ont pas indiqué leur sexe. Ces jeunes étudiaient en $4^{\mathrm{e}}$ année ( 45 soit $39 \%$ ), en $5^{\mathrm{e}}$ (48 soit $41 \%$ ) et en $6 \mathrm{e}$ année ( 24 soit $20 \%$ ), pour un total de 117 (un enfant n'a pas coché cet espace). Ils étaient âgés de 8 à 13 ans. Comme je n'avais pas demandé l'âge exact, les données sont présentées par catégories: un seul enfant avait entre 6 et 8 ans, alors que 62 avaient entre 9 et 10 ans et 55 , entre 11 et 13 ans.

Les enfants étaient originaires de 14 pays différents, outre le Canada (cinq élèves n'avaient pas identifié leur pays d'origine): trois d'Afrique, 79 d'Asie, 11 d'Amérique et deux d'Europe; 18 jeunes étaient d'origine canadienne et représentaient $15,3 \%$ de la cohorte étudiée. II faut noter ici que certains enfants étaient nés au Canada de mère et de père de nationalité autre que canadienne.

Puisque le pays d'origine constituait le critère de base de cette recherche, j'avais choisi l'école en fonction de ce critère. II n'est donc pas étonnant de constater que quatre-vingt-quinze répondants sur 118 étaient nés à l'extérieur du Canada. Le tableau 1 présente la répartition des jeunes selon le pays d'origine.

Tableau 1

Pays d'origine des enfants

\section{AFRIQUE}

Mozambique

Zaïre $\ldots \ldots \ldots \ldots \ldots \ldots$
Total .................

ASIE

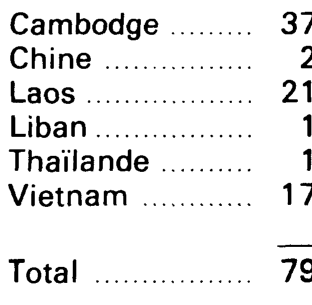

Sans réponse

\section{AMÉRIQUE}

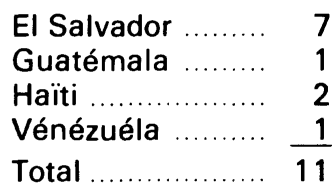

\section{EUROPE}

Hongrie ........... 1

Irlande $\ldots \ldots \ldots \ldots \ldots$
Total $\ldots \ldots \ldots \ldots \ldots \ldots \ldots$ $\frac{1}{11}$ . 年 
Les répondants parlaient douze langues différentes en plus du français et de l'anglais. Douze enfants $(12,2 \%)$ parlaient le français à la maison, un seul, l'anglais et quatre parlaient à la fois le français et l'anglais. Près de $86 \%$ des répondants parlaient une autre langue que le français et l'anglais chez eux; certains jeunes parlaient même plus d'une langue. La figure 1 illustre bien l'importance accordée à la conservation de la langue d'origine.

\section{Habitudes de lecture et intérêts particuliers}

La majorité des enfants, soit $56,7 \%$, ne lisaient pas dans la langue parlée à la maison (figure 2).
Tantôt, ils ne savaient pas lire cette langue (79\%), tantôt ils ne trouvaient pas de livres de cette langue $(7,5 \%)$ et, dans d'autres cas, cette lecture constituait un exercice trop difficile $(4,5 \%)$. II ne faut pas oublier que quelques enfants, qui avaient mentionné ne pas lire dans la ou les langue(s) parlée(s) à la maison, ont indiqué lire en français. Les langues que les jeunes ne lisaient pas sont principalement les langues vietnamienne, cambodgienne, espagnole, créole, chinoise et laotienne. Ainsi, des trente-sept enfants nés au Cambodge, vingt-huit $(75,7 \%)$ ne lisaient pas dans leur langue; des vingt et un répondants du Laos, seize $(75,2 \%)$ ne lisaient pas dans la langue parlée à la maison.

Fig. 1 - Répartition de la population étudiée en fonction de la langue parlée à la maison

Nombre

d'enfants

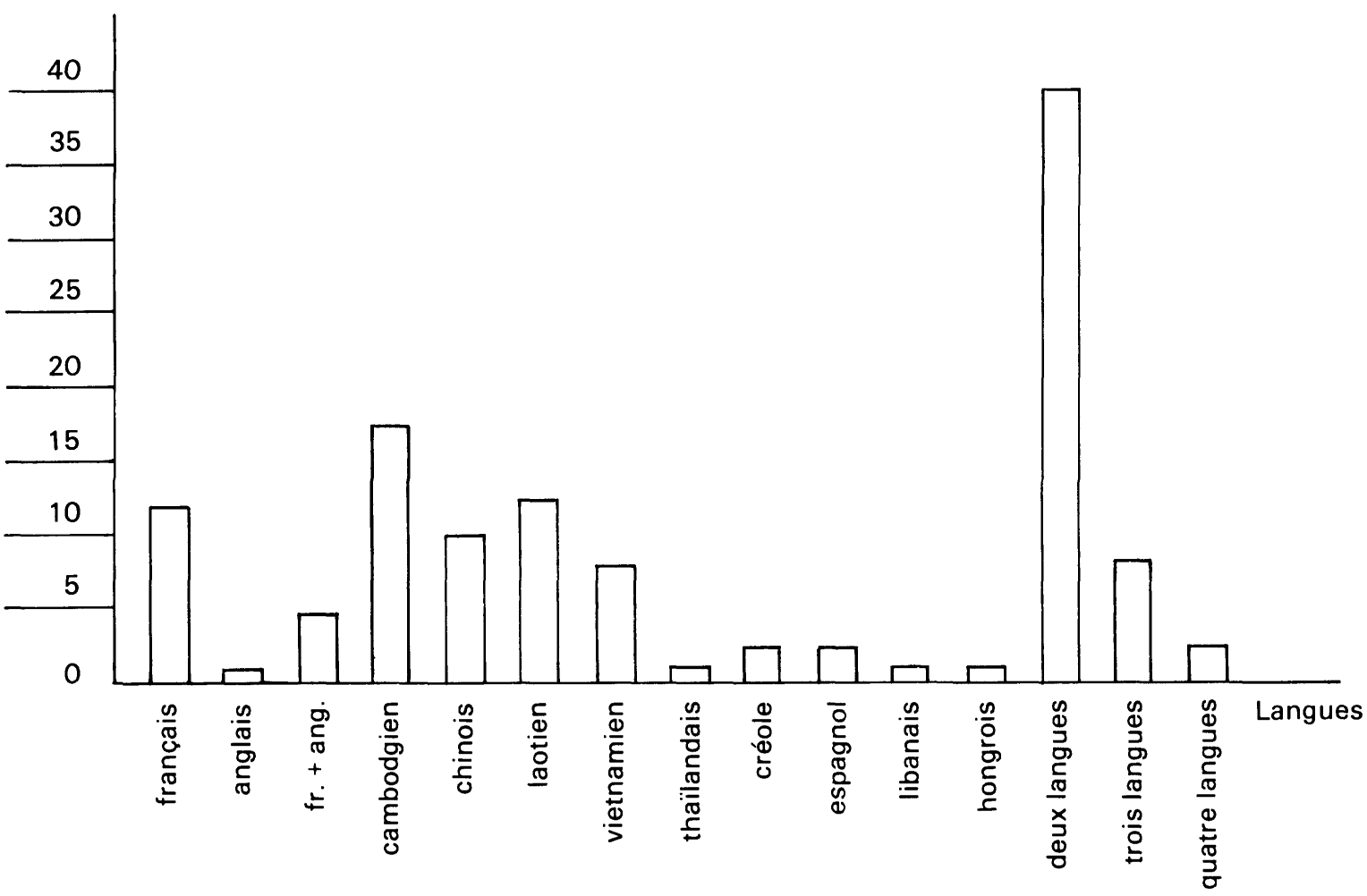


Par contre, quarante-neuf enfants, ou $42,3 \%$, lisaient dans leur langue maternelle. Ils se procuraient les livres de diverses façons: $17 \%$ par des échanges, $30 \%$ par l'achat et la majorité $(80,5 \%)$ par l'emprunt à la bibliothèque. Ces enfants étaient avant tout d'origine indochinoise $(25$ jeunes du Cambodge sur 27, 15 enfants nés au Vietnam sur 17,18 du Laos sur 21) et latino-américaine (cinq répondants du Salvador sur sept). Ils étaient aussi d'origine canadienne (13 enfants sur 18).
J'ai voulu identifier les activités domestiques qui intéressent le plus les enfants. Pour ce faire, j'ai établi huit catégories, plus une catégorie "autre» et j'ai demandé aux jeunes de pointer les activités qui occupaient une grande place parmi leurs loisirs. Comme l'indique le tableau 2, "regarder des vidéos" et "regarder la télévision " arrivent au premier et deuxième rang, suivis des activités «lire des livres" et "faire du sport».

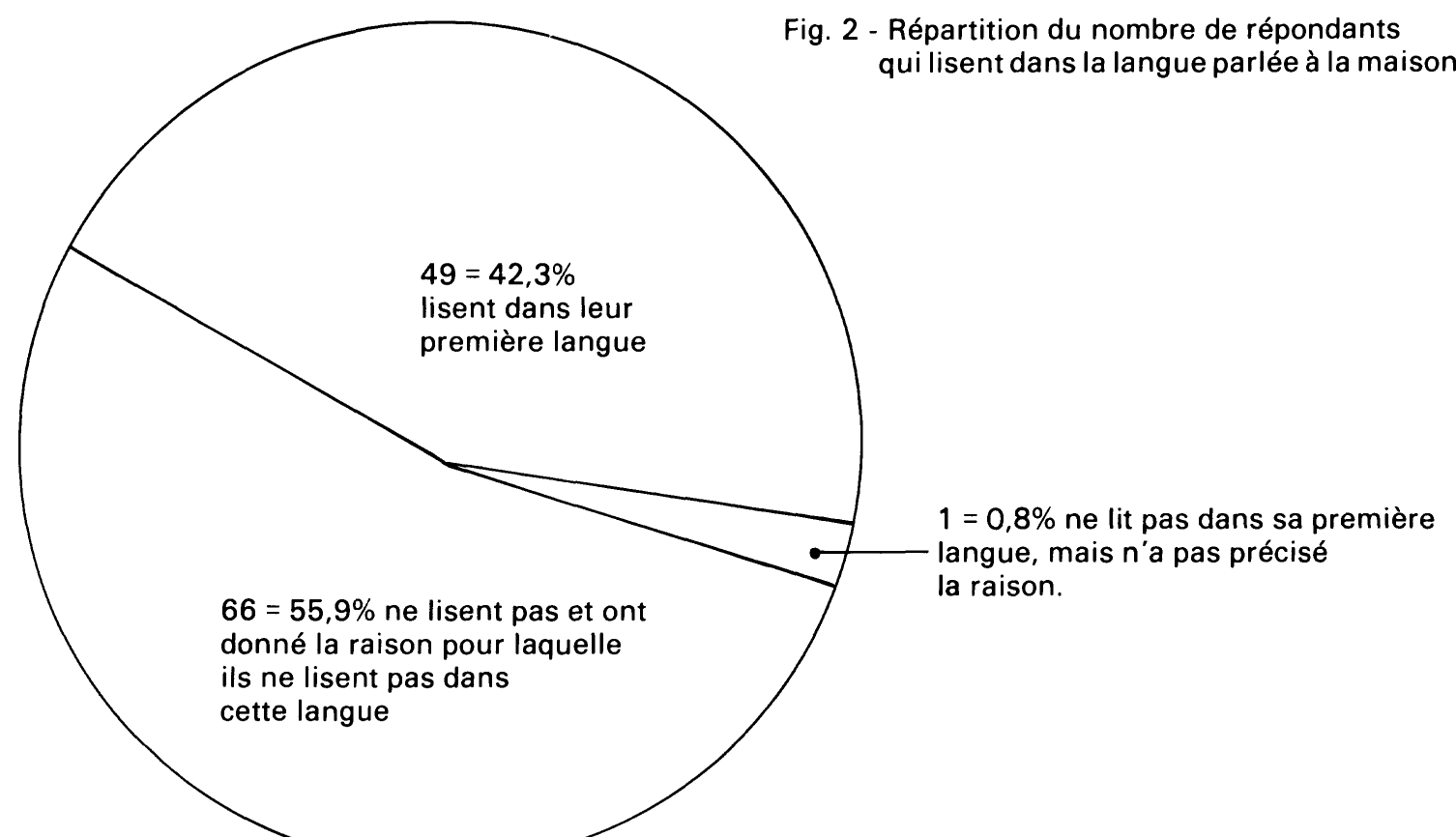

Note: 2 valeurs manquantes

Tableau 2

Activités préférées

\section{ACTIVITÉS}

regarder des vidéos

regarder la télévision

lire des livres

faire du sport

écouter des cassettes

jouer avec des amis et amies

lire des revues et des

bandes dessinées

écouter des disques

autre(s)

$\mathrm{N} \quad \%$

$67 \quad 56,7$

$58 \quad 49,2$

$55 \quad 46,6$

$51 \quad 43,2$

$46 \quad 38,9$

$45 \quad 38,1$

$42 \quad 35,6$

$29 \quad 24,5$

$32 \quad 27,1$
La question suivante portait sur les domaines d'intérêt des jeunes. Ici encore, j'ai établi des catégories, seize possibilités au total en plus du choix «autre». La compilation de ces données a permis de se faire une idée des habitudes de lecture des jeunes usagers, filles ou garçons. Le tableau 3 indique le nombre de réponses obtenues pour chaque catégorie d'ouvrages en fonction du pays d'origine. 


\section{Tableau 3}

\section{Sujets d'intérêt en fonction du pays d'origine}

\begin{tabular}{|c|c|c|c|c|c|c|c|c|c|c|c|c|c|c|c|c|c|}
\hline \multicolumn{18}{|l|}{ Sujets } \\
\hline 1. Sports & 6 & 24 & 1 & 14 & 0 & 7 & 0 & 1 & 1 & 2 & 1 & 2 & 1 & 0 & 1 & 61 & 517 \\
\hline 2. Ordinateurs & 11 & 18 & 0 & 10 & 1 & 10 & 0 & 1 & 0 & 2 & 0 & $\overline{1}$ & 0 & 0 & 1 & 55 & 46,6 \\
\hline 3. Animaux & 9 & 6 & 1 & 8 & 1 & 13 & 2 & 2 & 0 & 1 & 1 & 2 & 1 & 0 & 0 & 47 & 39,8 \\
\hline 4. Pays & 8 & 18 & 0 & 4 & 1 & 5 & 1 & 1 & 0 & 1 & 1 & 2 & 1 & 0 & 0 & 43 & 36,4 \\
\hline 5. Science & 7 & 14 & 0 & 5 & 0 & 8 & 1 & 1 & 0 & 2 & 0 & 2 & 1 & 0 & 0 & 41 & 34,7 \\
\hline 6. Musique & 8 & 8 & 0 & 9 & 1 & 6 & 1 & 1 & 1 & 3 & 0 & 1 & 1 & 0 & 1 & 41 & 34,7 \\
\hline 7. Nature & 6 & 17 & 0 & 3 & 0 & 7 & 0 & 1 & 1 & 2 & 1 & 2 & 1 & 0 & 0 & 41 & 34,7 \\
\hline 8. Extra-terrestres & 6 & 9 & 0 & 4 & 0 & 4 & 2 & 2 & 0 & 2 & 1 & 2 & 1 & 1 & 0 & 34 & 28,8 \\
\hline 9. Artisanat* & 4 & 10 & 0 & 6 & 0 & 6 & 0 & 0 & 1 & 3 & 1 & 0 & 0 & 0 & 0 & 31 & 26,2 \\
\hline 10. Avions & 2 & 8 & 1 & 6 & 1 & 5 & 1 & 2 & 0 & 0 & 0 & 1 & 1 & 0 & 0 & 28 & 23,7 \\
\hline 11. Légendes & 6 & 5 & 0 & 8 & 0 & 8 & 0 & 0 & 0 & 0 & 1 & 0 & 0 & 0 & 0 & 28 & 23,7 \\
\hline 12. Biographies & 6 & 5 & 0 & 3 & 0 & 6 & 1 & 0 & 0 & 1 & 0 & 0 & 0 & 0 & 0 & 22 & 18,6 \\
\hline 13. Danse & 5 & 3 & 0 & 2 & 0 & 1 & 1 & 0 & 1 & 5 & 1 & 1 & 1 & 0 & 1 & 22 & 18,6 \\
\hline 14. Bateaux & 2 & 5 & 0 & 0 & 0 & 4 & 1 & 1 & 0 & 1 & 0 & 0 & 0 & 0 & 0 & 14 & 11,9 \\
\hline 15. Automobiles & 1 & 4 & 0 & 1 & 0 & 3 & 1 & 1 & 0 & 0 & 0 & 0 & 0 & 0 & 1 & 12 & 10,2 \\
\hline 16. Trains & 1 & 6 & 0 & 0 & 0 & 3 & 1 & 1 & 0 & 0 & 0 & 0 & 0 & 0 & 0 & 12 & 10,2 \\
\hline 17. Autres & & & & & & & & & & & & & & & & 3 & 2,5 \\
\hline chimie & 1 & & & & & & & & & & & & & & & & \\
\hline $\begin{array}{l}\text { mécanique } \\
\text { robots }\end{array}$ & & & & 1 & & & 1 & & & & & & & & & & \\
\hline
\end{tabular}

* Cette catégorie comprenait le macramé, la poterie, le tricot, la cuisine, les arts plastiques, la couture, la menuiserie et la peinture.

\section{Tableau 4}

Taux de fréquentation de la bibliothèque municipale en fonction du pays d'origine

\begin{tabular}{lccccccc} 
PAYS & N. DE REP & SOUVENT/ & A L'OCCASION & TOTAL & $\%$ & JAMAIS & 1 FOIS AVEC LA CLASSE \\
\hline Canada & 18 & 2 & 7 & 9 & 50 & 2 & 7 \\
Cambodge & 37 & 12 & 16 & 28 & 75,6 & 6 & 3 \\
Chine & 2 & 1 & 0 & 1 & 50 & 1 & 0 \\
Laos & 21 & 5 & 13 & 18 & 85,7 & 2 & 1 \\
Liban & 1 & 0 & 1 & 1 & 100 & 0 & 0 \\
Thaïlande & 1 & 1 & 0 & 1 & 100 & 0 & 3 \\
Vietnam & 17 & 3 & 9 & 12 & 70 & 2 & 0 \\
Guatémala & 1 & 1 & 0 & 1 & 100 & 0 & 3 \\
El Salvador & 7 & 1 & 2 & 3 & 42,8 & 1 & 0 \\
Haïti & 2 & 0 & 2 & 2 & 100 & 0 & 0 \\
Vénézuéla & 1 & 1 & 0 & 1 & 100 & 0 & 0 \\
Mozambique & 2 & 1 & 1 & 2 & 100 & 0 & 0 \\
Zaïre & 1 & 1 & 0 & 0 & 0 & 0 & 0 \\
Hongrie & 1 & 1 & 1 & 1 & 100 & 0 & 1 \\
Irlande & 1 & 1 & 0 & 1 & 100 & 0 & 0 \\
TOTAL & $113^{*}$ & 29 & 52 & 81 & 71,6 & 14 & 18 \\
\hline
\end{tabular}




\section{Les bibliothèques municipale et scolaire}

Cette section du questionnaire était consacrée à l'évaluation de la collection et des services offerts aux deux bibliothèques étudiées: taux de fréquentation (souvent, à l'occasion, jamais, avec la classe), raison d'utilisation de la bibliothèque municipale (travaux scolaires, lecture de détente, rencontre d'amis, activités spéciales), raisons d'ignorer la bibliothèque municipale et comparaison des deux bibliothèques.

Les enfants vont-ils à la Bibliothèque municipale de Saint-Laurent? Près de $72 \%$ des répondants s'y rendaient régulièrement ou à l'occasion. Le tableau 4 démontre clairement que la bibliothèque, au sens large, répond à un besoin de lecture.

Seulement quinze jeunes (14 sur le tableau 4) ne fréquentaient pas la bibliothèque municipale parce qu'ils n'avaient pas le temps ou encore parce qu'elle est située trop loin de chez eux. Les autres y allaient surtout pour emprunter des livres de détente $(72,8 \%)$ et pour compléter des travaux scolaires $(31,1 \%)$. Seulement quinze abonnés participaient aux activités spéciales et douze répondants disent se rendre à la bibliothèque pour fraterniser avec des amis et amies.

Dans $94 \%$ des cas, les jeunes trouvaient les livres qu'ils cherchaient à la bibliothèque municipale et aimaient les livres qu'ils choisissaient. Les enfants étaient aussi très satisfaits de leur bibliothèque scolaire. Plus de $90 \%$ d'entre eux la trouvaient confortable et accueillante; $70 \%$ lisaient toujours les livres empruntés et $54 \%$ les aimaient toujours.

Les deux bibliothèques ont su s'adapter aux goûts des jeunes de sorte qu'ils s'y sentent à l'aise. II n'est donc pas surprenant que les opinions soient partagées sur l'endroit où les enfants se sentent le plus à l'aise: $24,5 \%$ ont répondu à la bibliothèque municipale, $24,5 \%$ à la bibliothèque de l'école et $25,4 \%$ aux deux endroits sans préférence. Quelle que soit leur nationalité, les enfants conçoivent la bibliothèque comme un endroit où il fait bon lire et apprendre. On la perçoit comme un lieu de savoir. Peu d'élèves se prévalaient des activités spéciales telles que le club de lecture et les ateliers de la bibliothèque municipale.

Les enfants étaient appelés aussi à indiquer les types d'ouvrages qu'ils préféraient entre les contes, les revues, les romans, les bandes dessinées, les livres d'information ou documentaires. Les bandes dessinées remportent cette épreuve haut la main avec $62 \%$ des répondants, sans distinction de sexe ou de pays d'origine. Suivent les contes (49\%), les livres d'information $(27,1 \%)$, les romans $(15,2 \%)$ et les revues $(13,5 \%)$. Ces résultats ne sont pas étonnants dans le contexte québécois et à l'ère des jeux vidéos, des vidéos et de la télévision. Plusieurs enfants préfèrent voir une image (puisqu'une image vaut mille mots) sur papier plutôt que de s'en faire une eux-mêmes à partir d'un texte.
La question 19 offrait l'occasion aux jeunes d'indiquer la ou les langue(s) désirées. Ils avaient le choix entre l'espagnol, le cambodgien, le vietnamien, le chinois, le laotien, le créole et autre(s). Comme l'on pouvait s'y attendre à la suite des résultats obtenus à la question 3 , le cambodgien est la langue la plus désirée (par trente-deux répondants, soit $27,2 \%$ ), suivie du chinois, du vietnamien, du laotien, de l'espagnol et du créole. Cet ordre est fonction du pays d'origine des enfants et du nombre de répondants de chaque catégorie. Plusieurs jeunes nés au Canada, au Québec plus particulièrement, s'intéressaient aux langues étrangères. Sur dix-huit répondants, sept s'intéressaient au créole, six à l'espagnol, quatre au chinois, trois au cambodgien et un au vietnamien. Le tableau 5 donne un aperçu de ces résultats.

\section{Tableau 5}

\section{Langues désirées}

\begin{tabular}{lc} 
LANGUES & NOMBRE \\
\hline cambodgien & 32 \\
chinois & 30 \\
vietnamien & 21 \\
laotien & 19 \\
espagnol & 17 \\
créole & 14 \\
autres & 10 \\
thaïlandais & 2 \\
arabe & 1 \\
hindi & 1 \\
hongrois & 1 \\
irlandais & 1 \\
italien & 1 \\
lingala* & 1 \\
portugais & 1 \\
ukrainien & 1
\end{tabular}

* langue africaine

La question 20 laissait place à l'imagination de l'enfant. Certains ont écrit de petites oeuvres d'art, une chanson et un poème, en guise de commentaire. Au total, trente-neuf enfants ont rempli cette partie du questionnaire. En plus des commentaires écrits, les jeunes ont posé des questions oralement afin de savoir ce qu'il faut faire lorsqu'on a perdu sa carte, ce qu'il en coûte pour s'en faire faire une autre, des documents dont ils ont besoin pour l'abonnement.

Sur les trente-neuf commentaires, neufs portaient sur le questionnaire. Seize commentaires étaient d'ordre général (félicitations, remerciements, etc.); huit portaient sur les bibliothèques au sens large et six concernaient la Bibliothèque municipale de Saint-Laurent et la bibliothèque de l'école. Au sujet de la bibliothèque municipale, deux enfants ont écrit que la bibliothèque est 
calme et qu'il y a beaucoup de livres intéressants; un autre a précisé qu'il y avait plus de livres et de variété qu'à la bibliothèque de l'école. Un enfant espère avoir un jour autant de livres qu'il y en a à la bibliothèque municipale! Les commentaires généraux démontrent l'intérêt des enfants pour la découverte et le désir de connaître. Un jeune aime bien aller à l'école parce que cette dernière est située près de sa maison. Un autre aimerait qu'ily ait un vidéo à la bibliothèque tandis qu'un troisième aimerait y voir un poste de télévision. D'autres écoliers aiment la bibliothèque parce qu'il y a de belles histoires, parce que cela fait connaître plus de choses sur l'univers et sur la vie dans les autres pays. Enfin un répondant a ajouté vouloir des livres dans sa langue (le cambodgien) parce qu'il veut apprendre à lire et à écrire dans cette langue.

Comme on peut le voir, les problèmes reliés à l'intégration et à la diversité culturelle sont loins d'être solutionnés. Au cours de la recherche, j'ai appris que, dans le passé, la Bibliothèque de Saint-Laurent avait offert aux enfants des livres en langues étrangères. En raison du nombre peu élevé d'emprunts de livres pour enfants à la section des jeunes puis à la section des adultes, le service a été annulé et on s'est concentré sur les livres en langues étrangères pour adultes, d'ailleurs très populaires. Le service pourrait être remis sur pied si la demande le justifiait. Je crois que la Bibliothèque municipale de Saint-Laurent pourrait tirer profit de la collection pour enfants du Biblioservice multilingue de la Bibliothèque nationale du Canada et que cette collection serait utilisée à bon escient si le service était annoncé aux endroits appropriés (écoles, CLSC, centres de loisirs, églises, groupes multi-ethniques, etc.). Ville Saint-Laurent, comme on l'a déjà dit, compte une quarantaine de groupes ethniques. L'établissement de relations étroites avec ces groupes et associations constitue un excellent moyen de connaître la population et de se faire connaître auprès d'elle. Les deux parties pourraient profiter de ces échanges: la bibliothèque publique en se familiarisant avec le mode de vie de ces nouveaux usagers et en s'appuyant sur eux pour démontrer la nécessité du Biblioservice multi-lingue. Ces personnes pourraient même contribuer au choix et à l'acquisition de volumes en langues étrangères. Les jeunes abonnés bénéficieraient de ce service, car il leur donnerait l'occasion de rester en contact avec leur langue maternelle.

Tout en sauvegardant l'orientation française de l'école, l'achat de livres bilingues (français et une autre langue) constitue un compromis que les jeunes apprécieraient.

Les jeunes néo-québécois auraient donc les mêmes intérêts et les mêmes besoins que les enfants d'origine québécoise et canadienne. Le plus difficile n'est pas d'identifier les livres qui leur plaisent mais de trouver ceux qui leur permettraient de conserver leur patrimoine culturel et linguistique.

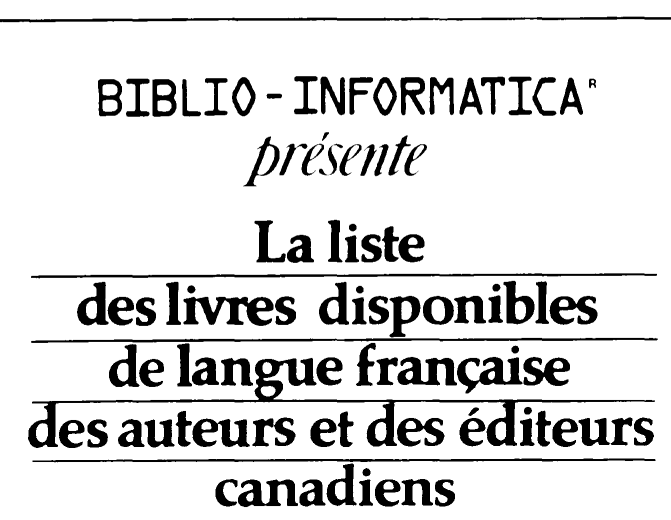

Canadian

Authors \& Publishers

French Books in Print

Un outil de commercialisation et de référence unique!

Plus de

20,000 titres 439 éditeurs 260 distributeurs Enfin à votre disposition, une banque de données originale, pratique et facile à consulter présentant toute la production canadienne de langue trançalse.

\section{on 3 romence Auteurs •Titres•Sujets}

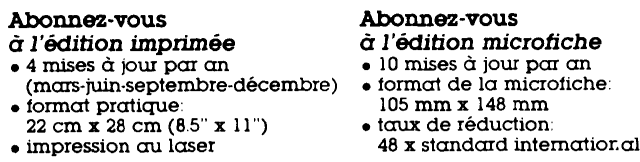

Abonnez-vous - 10 mises à jour par an - format de la microfiche: $105 \mathrm{~mm} \times 148 \mathrm{~mm}$ $48 \times$ standard internatior. $\alpha$

Renseignement et abonnements:
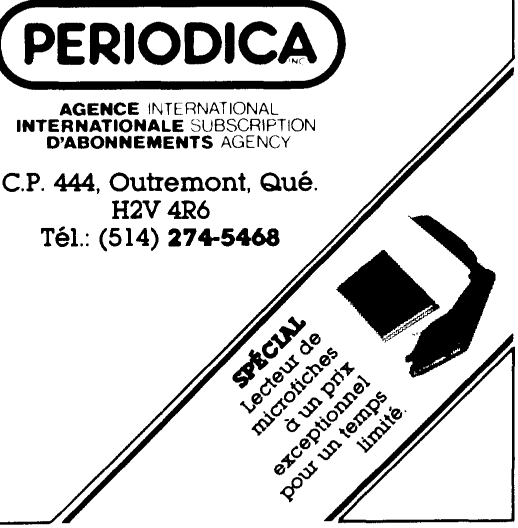